\title{
Second generation HIV surveillance in Pakistan: evidence for understanding the epidemic and planning a response
}

\author{
James F Blanchard, ${ }^{1}$ Laura H Thompson, ${ }^{1}$ Sevgi 0 Aral ${ }^{2}$
}

More than a decade ago UNAIDS and the WHO published a set of guidelines for the conduct of what was termed 'secondgeneration HIV surveillance', which encouraged the enhancement of HIV surveillance to provide better information about the patterns, status and trajectory of the HIV epidemic to better inform policies and programmes. ${ }^{1}$ As Rehle and colleagues have noted, key aspects of the second-generation approach include: combining information from different components of surveillance to achieve higher degrees of explanatory power, targeting segments of the population in which most new HIV infections are occurring; and integrating surveillance based on biological markers of infection (ie, HIV serosurveillance) with behavioural data. ${ }^{2}$ Since then, second-generation surveillance has become widely used in diverse global contexts, with substantial emphasis on two key components. The first component, sentinel HIV surveillance, has been conducted at convenient venues to track the amplitude and trends of HIV prevalence among populations based on presumed stratification of risk, such as women attending antenatal clinics, men and women attending public sexually transmitted disease clinics, and key populations at higher risk (such as female sex workers) receiving HIV prevention services. While this approach has provided important insights into the distribution and trends of HIV in different population segments, it has substantial constraints. Perhaps most importantly, it sacrifices representativeness for sampling efficiency, leading to uncertainty as to how well the results truly reflect the HIV prevalence in these populations. In addition, sentinel surveillance does not incorporate any assessment of behavioural trends, or the linkage

\footnotetext{
${ }^{1}$ Department of Community Health Sciences, Centre for Global Public Health, University of Manitoba, Winnipeg, Canada; ${ }^{2}$ Division of STD Prevention, Centers for Disease Control and Prevention, Atlanta, USA

Correspondence to Dr James F Blanchard, Department of Community Health Sciences, Centre for Global Public Health, University of Manitoba, R070-771 McDermot Ave, Winnipeg, Canada R3E OT6; james_blanchard@umanitoba.ca
}

between biological, socio-demographic, and behavioural variables with HIV status. As a result, it does not provide information about patterns and trends in the key behaviours that influence epidemics, nor does it permit a more in-depth understanding of relationships between socio-demographic characteristics and behaviours with HIV prevalence. To address this deficit, the second main component developed by second-generation surveillance systems have been integrated bio-behavioural surveys (IBBS), particularly for key populations such as female sex workers (FSWs), injecting drug users (IDUs) and high risk men who have sex with men (MSM). The IBBS has become a core component of secondgeneration HIV surveillance in concentrated HIV epidemics since it provides policy makers and programme planners with information about the behavioural characteristics and trends in key populations, along with knowledge about how these parameters influence the trajectory of HIV epidemics.

Mounting a national second-generation HIV surveillance programme is a challenging technical and managerial task, requiring sustained high level commitment and resource mobilisation. It demands a high level of technical capacity to design the various data collection components, highly competent field-level implementation capacity to collect biological and behavioural data from key populations, and advanced capacity for data management, analysis, and translation to ensure that data is communicated effectively to policy makers, programme planners and implementers, and key populations. In this light, the second-generation HIV surveillance programme conducted in Pakistan and described in this Supplement is a notable accomplishment, and a strong endorsement for the feasibility and utility of this approach.

There are several aspects of Pakistan's second-generation surveillance system that merit specific mention. First, the system was established and embedded as an early cornerstone of an enhanced HIV response, identified as a specific component with a specific resource allocation within the national strategy. ${ }^{3}$ This enabled the development of a national platform for the surveillance system, along with a core technical capacity and infrastructure for the design, implementation, data management and analysis, and translation and dissemination of results. Second, the programme was specifically designed for the concentrated nature of Pakistan's epidemic, therefore focusing resources on surveillance in key populations, including FSWs, IDUs, and male and hijra (transgender) sex workers. Third, although funded externally, the implementation of key aspects of the surveillance was largely conducted through existing technical/ research institutions and non-government organisations, thereby creating a substantial pool of expertise within Pakistan for carrying out the field operations and other key processes.

Another important accomplishment of Pakistan's second-generation HIV surveillance was the development and wide application of a standardised method for mapping the key populations. As illustrated in articles in this supplement, there are several compelling reasons for incorporating the mapping of key populations as a standard component of all secondgeneration surveillance systems. First, mapping provides crucial information about the size, distribution, and characteristics of key populations, and how this is changing over time. The size of key populations such as FSWs, IDUs, and male and hijra sex workers is a necessary input into models that estimate their relative contribution to HIV transmission dynamics, but in many jurisdictions these population estimates are based on standardised assumptions about the relative size of these populations (ie, as a percent of the adult population). As illustrated in the article by Reza and colleagues in this Supplement, mapping in Pakistan showed substantial differences in the relative size and potential contribution of FSWs, IDUs, and male and hijra sex workers to the HIV epidemics in different cities. ${ }^{4}$ Estimating the size of the key populations is also important for estimating the resources needed for HIV prevention programmes, and how they should be distributed to achieve allocative efficiency as illustrated in the article by Emmanuel et $a .^{5}$ Another key finding from the mapping in Pakistan is how the typologies of FSWs are changing over time, which provides important guidance for programme planners and implementers. Another important benefit of mapping is that it provides the basis for more representative sampling of key populations for bio-behavioural surveys by defining a 
sampling frame. In many jurisdictions, sampling is still largely based on known locations of key populations identified by existing prevention programmes. This often results in oversampling of key populations already reached by programmes and the biases inherent in such samples. The very low proportion of key populations sampled in Pakistan's bio-behavioural surveys that knew of or utilised services provided through service delivery programmes illustrates how successful the mapping was at delivering a sample frame unbiased by the reach of existing programmes. Moreover, it permitted an assessment of trends in service coverage. For example, the overall proportion of FSWs who had accessed HIV prevention programmes increased from 2.1\% in 2006 to $20.7 \%$ in $2011 .^{6}$

The articles assembled in this Supplement provide important insights into the emerging HIV epidemics in Pakistan. The article by Reza et al. provides wide-ranging analysis of the absolute and relative sizes of key populations in different cities of Pakistan, overlapping networks between the key populations, and the implications for HIV transmission dynamics. ${ }^{4}$ They found that the relative size of key populations varied widely between cities, as did the overlap between high risk sexual and drug injecting networks. Another key finding was that, as anticipated, HIV epidemics among IDUs have emerged most rapidly and IDUs will continue to have the highest HIV prevalence. However, projection models indicate an increasing risk of substantial expansion of the HIV epidemics in high risk sexual networks, particular among male and hijra (transgender) sex workers, and eventually among female sex workers. The article by Emmanuel et al. examines the size and distribution of the key populations in different cities of Pakistan and analyses how HIV prevention programme resources could be allocated to most efficiently achieve high programme coverage. $^{5}$

The next four articles provide detailed analysis of the patterns and trends in the behaviours, HIV prevalence, and associations between socio-demographic and behavioural factors and HIV risk among key populations. Archibald et al. show the rapid expansion of HIV prevalence among IDUs in Pakistan, illustrating the importance of intensified prevention efforts. ${ }^{7}$ They also illustrate how IDU risk differs geographically, with varying injecting patterns and trends in HIV prevalence in different cities. The next article, by Emmanuel and colleagues, illustrates the great variability in female sex work in different cities of Pakistan, both in terms of the relative size of the FSW population and the typology of sex work. ${ }^{8}$ They also show how female sex work in Pakistan has been changing, with an increasing dispersion of sex work into forms that are mediated through network operators, facilitated with the increasing use of cell phones by FSWs to interact with clients. The article by Mishra and colleagues described the socio-demographic and behavioural characteristics of FSWs, and how they have changed between 2006 and 2011. ${ }^{6}$ Key findings include low levels of HIV prevention programme exposure and low rates of condom use. Notably, FSWs who had experienced sexual violence and had recent sexual relations with a person who injects drugs were less likely to use condoms. Thompson and colleagues provide a comprehensive description of the male and hijra (transgender) sex worker population in different regions of Pakistan. 9 They show the variation in these populations across the major regions with respect to the social organisation of sex work. Across all cities, these populations experience very high risk for HIV, and variable knowledge and access to service delivery programmes.

The final two papers describe management and policy issues experienced in Pakistan in the context of the secondgeneration HIV surveillance programme. Munro et $\mathrm{al}^{3}$ provide a broad-ranging analysis of the policy challenges and opportunities related to HIV surveillance in Pakistan. They highlight the implementation challenges, which relate to the changing funding and programme environment, requiring flexibility in implementation methods while retaining methodological consistency and rigour. They note the ongoing challenges of ensuring that surveillance activities maintain high ethical standards, particularly since they often involve highly vulnerable populations that are marginalised and stigmatised. They also point out the importance of high level commitment to surveillance and the translation of findings into policy and programme design as key to the sustainability of second-generation surveillance. Finally, Adrien and colleagues examine how the lessons from Pakistan's second-generation surveillance programme can be translated to other contexts. ${ }^{10}$ They conclude that many of the technical strategies developed and deployed in Pakistan are applicable in diverse contexts, including both developed and developing countries. They also emphasise the need for continuous interaction between those conducting surveillance and those responsible for HIV prevention programme management and policy to ensure efficient and effective knowledge translation to improve programme design and implementation.

Globally, there is an increasing emphasis on improving the efficiency and effectiveness of HIV prevention programmes. Achieving this will require high quality methods to appraise epidemics and translate those appraisals into strategic plans that will effectively direct HIV prevention resources and interventions to where they will have the greatest impact. The articles in this Supplement present a comprehensive set of analyses which form the 'assessment' and 'strategic planning' components of a programme science approach to HIV prevention programme planning. ${ }^{11} 12$ This also requires the ongoing monitoring of the results of programmes, while simultaneously tracking changes in the epidemics. In addition, as illustrated in this collection of articles, it is important to understand the characteristics of the key populations, and how they are changing over time in response to shifting social, economic and political contexts. Pakistan's secondgeneration HIV surveillance programme illustrates some key methods for doing this, and could serve as a model for other countries seeking evidence-based HIV programmes.

Contributors JFB, LHT, and SA co-wrote this Editorial.

Funding Canadian International Development Agency. Competing interests None.

Provenance and peer review Commissioned; internally peer reviewed.
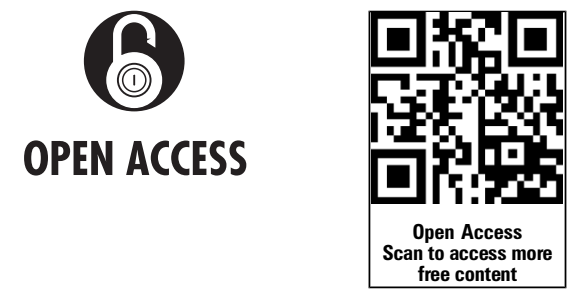

Open Access This is an Open Access article distributed in accordance with the Creative Commons Attribution Non Commercial (CC BY-NC 3.0) license, which permits others to distribute, remix, adapt, build upon this work non-commercially, and license their derivative works on different terms, provided the original work is properly cited and the use is non-commercial. See: http:// creativecommons.org/licenses/by-nc/3.0/

To cite Blanchard JF, Thompson LH, Aral SO. Sex Transm Infect 2013;89:ii1-ii3.

Received 23 July 2013

Accepted 28 July 2013

Sex Transm Infect 2013;89:ii1-ii3.

doi:10.1136/sextrans-2013-051297 


\section{REFERENCES}

1 Guidelines for second-generation HIV surveillance. Geneva: UNAIDS/WHO; 2000. UNAIDS/WHO document WHO/CDS/CSR/EDC/2000.5, UNAIDS/00.03E.

2 Rehle T, Lazzari S, Dallabetta G, et al. Second-generation HIV surveillance: better data for decision-making. Bull WHO 2004;82:121-7.

3 Munro M, Holte-McKenzie M, Ahmed S, et al. Second generation HIV surveillance in Pakistan: policy challenges and opportunities. Sex Transm Infect 2013;89:ii48-52.

4 Reza T, Melesse DY, Shafer LA, et al. Patterns and trends in Pakistan's heterogeneous HIV epidemic. Sex Transm Infect 2013;89:ii4-10.

5 Emmanuel F, Thompson LH, Salim M, et al. The size and distribution of key populations at greater risk of
HIV in Pakistan: implications for resource allocation for scaling up HIV prevention programmes. Sex Transm Infect 2013;89:ii11-7.

6 Mishra S, Thompson LH, Sonia A, et al. Sexua behaviour, structural vulnerabilities and HIV prevalence among female sex workers in Pakistan. Sex Transm Infect 2013;89:ii34-42.

7 Archibald CP, Shaw SY, Emmanuel F, et al. Geographical and temporal variation of injection drug users in Pakistan. Sex Transm Infect 2013;89:ii18-28.

8 Emmanuel F, Thompson LH, Athar U, et al. The organisation, operational dynamics and structure of female sex work in Pakistan. Sex Transm Infect 2013;89:ii29-33.

9 Thompson LH, Salim M, Baloch CR, et al. Heterogeneity of characteristics, structure, and dynamics of male and hijra sex workers in selected cities of Pakistan. Sex Transm Infect 2013;89: ii43-7.

10 Adrien A, Thompson LH, Archibald CP, et al. Translating knowledge from Pakistan's second generation surveillance system to other global contexts. Sex Transm Infect 2013:89:ii53-7.

11 Blanchard JF, Aral SO. Program Science: an initiative to improve the planning, implementation and evaluation of HIV/sexually transmitted infection prevention programmes. Sex Transm Infect 2011; 87:2-3.

12 Aral SO, Blanchard JF. The Program Science initiative: improving the planning, implementation and evaluation of HIV/STI prevention programs. Sex Transm Infect 2012;88:157-9. 